\title{
OPTIMIZING DISTANCE CONSTRAINTS FREQUENCY ASSIGNMENT WITH RELAXATION
}

\author{
Zehui ShaO ${ }^{1}$, Enqiang Zhu ${ }^{1}$, Jin Xu², Aleksander Vesel ${ }^{3, *}$ and Xiujun Zhang ${ }^{4}$
}

\begin{abstract}
In a typical wireless telecommunication network, a large number of communication links is established with a limited number of available frequencies. The problem that addresses assigning available frequencies to transmitters such that interference is avoided as far as possible is called the frequency assignment problem. The problem is usually modeled as a graph coloring (labeling) problem. We study in this paper the $(s, t)$-relaxed $L(2,1)$-labeling of a graph which considers the situation where transceivers that are very close receive frequencies that differ by at least two while transceivers that are close receive frequencies that differ by at least one. In addition, the model allows at most $s$ (resp. $t$ ) anomalies at distance one (resp. two). The objective of the model is to minimize the span of frequencies in a corresponding network. We show that it is NP-complete to decide whether the minimal span of a $(1,0)$-relaxed $L(2,1)$-labeling of a graph is at most $k$. We also prove that the minimal span of this labeling for two classes of graphs is bounded above with the the square of the largest degree in the graph of interest. These results confirm Conjecture 6 and partially confirm Conjecture 3 stated in Lin [J. Comb. Optim. 31 (2016) 1-22].
\end{abstract}

Mathematics Subject Classification. 05C15, 05C90.

Received February 12, 2018. Accepted April 28, 2020.

\section{INTRODUCTION}

In a wireless telecommunication network, communication connections among transmitters are established by means of radio links. A national regulation authority assigns for a wireless network provider a certain frequency spectrum which is divided into so-called channels. The number of transmitters in a typical telecommunication network is much larger than the number of available channels, therefore some of the transmitters have to use same channels. However, the use of channels is limited by interference which can disable communication by means of transmitted signals. Interference depends on a lot of factors, the most important of which is the distance between two transmitters. In other words, if a transmitter and its close neighbor use the same channel, the result can be a failure of the data transmission. Moreover, interference can also occur if two transmitters that are very close use adjacent channels.

\footnotetext{
Keywords. Frequency assignment, $(s, t)$-relaxed $L(2,1)$-labeling, $L(2,1)$-labeling.

1 Institute of Computing Science and Technology, Guangzhou University, Guangzhou 510006, P.R. China.

2 School of Electronic Engineering and Computer Science, Peking University, Beijing 100871, P.R. China.

3 Faculty of Natural Sciences and Mathematics, University of Maribor, Koroška cesta 160, Maribor 2000, Slovenia.

4 School of Information Science and Engineering, Chengdu University, Chengdu 610106, P.R. China.

${ }^{*}$ Corresponding author: aleksander.vesel@um.si
} 
For the reasons described above, the restricted availability of frequencies is one of the most important problems facing the radio network operators today. The problem that addresses assigning available frequencies to transmitters such that interference is avoided as far as possible is called the Frequency Assignment Problem (FAP).

A natural way to approach this problem is to model the described situation with a graph. A detailed graph theoretical models of FAP is the concept of distance constrained labeling of graphs [7]. The most examined variant of this concept is the $L(2,1)$-labeling problem where adjacent vertices must be assigned labels of distance at least two apart and vertices of distance two must be assigned different labels [5].

As usual, the distance between vertices $u$ and $v$ of a graph $G$ is the shortest path distance and will be denoted by $d(u, v)$. A vertex $v$ of a graph $G$ is a neighbor (resp. a 2-neighbor) of $u$ if $u$ is adjacent to $v$ (resp. at distance two from $v$ ) in $G$.

An $L(2,1)$-labeling of a graph $G$ is a function $f$ from the vertex set $V(G)$ to the set of nonnegative integers such that

(1) $f(u)-f(v) \notin\{0,-1,1\}$ if $u$ and $v$ are neighbors;

(2) $f(u) \neq f(v)$ if $u$ and $v$ are 2-neighbors.

The span of $f$ is the difference between the largest and the smallest number in $f(V(G))$. The $\lambda$-number of $G$, denoted by $\lambda(G)$, is the minimum span over all $L(2,1)$-labelings of $G$.

Note that for the path, cycle and complete graph on $n$ vertices we have $\lambda\left(P_{n}\right) \leq 4, \lambda\left(C_{n}\right)=4$ and $\lambda\left(K_{n}\right)=$ $2(n-1)$, respectively [5].

Since the spectrum of frequencies is very limited, there may not exist an $L(2,1)$-labeling of a given graph with a requested number of labels. Motivated with this situation, Lin [8] proposed the $(s, t)$-relaxed $L(2,1)$-labeling (see also $[1,2,13])$.

For nonnegative integers $s, t$ and $k$, an $(s, t)$-relaxed $k-L(2,1)$-labeling $f$ of a graph $G$ is an assignment of labels from $\{0,1, \cdots, k\}$ to the vertices of $G$ if the following three conditions are satisfied:

(1) $f(u) \neq f(v)$ if $u$ and $v$ are neighbors;

(2) for any vertex $u$ of $V(G)$, there are at most $s$ neighbors of $u$ receiving labels from $\{f(u)-1, f(u)+1\}$;

(3) for any vertex $u$ of $V(G)$, the number of 2-neighbors of $u$ with the label $f(u)$ is at most $t$.

The $(s, t)$-relaxed $L(2,1)$-labeling number $\lambda_{2,1}^{s, t}(G)$ of $G$ is the minimum $k$ such that $G$ admits an $(s, t)$-relaxed $k$ - $L(2,1)$-labeling.

If $f$ is an $(s, t)$-relaxed $L(2,1)$-labeling, then the difference between the maximum and minimum labels used under $f$ is denoted as $\operatorname{span}(f)$.

In [8], the following problem is proposed:

Problem 1: Fixed parameter relaxed $L(2,1)$-labeling (FPRL21).

Instance: Graph $G=(V, E)$ and a nonnegative integer $k$.

Question: Is $\lambda_{2,1}^{1,0}(G) \leq k$ ?

Conjecture 1.1 ([8]). FPRL21 is NP-complete for every $k \geq 3$.

They also suggested the following weak version of Griggs and Yehs conjecture:

Conjecture $1.2([8])$. For any graph $G$ with maximum degree $\Delta, \lambda_{2,1}^{1,0}(G) \leq \Delta^{2}$.

Lattices (also called grids) are frequently used models for the frequency assignment problems. In particular, the $(s, t)$-relaxed $L(2,1)$-labeling number of the square lattice is determined in [1], the $(s, t)$-relaxed $L(2,1)$ labeling number of the triangular lattice in [2], while the $(s, t)$-relaxed $L(2,1)$-labeling number of the hexagonal lattice is given in [3].

The paper is organized as follows. In the sequel of this section we give basic definitions needed in this paper. In Section 2, we confirm Conjecture 1.1 by showing that it is NP-complete to decide whether $\lambda_{2,1}^{1,0}(G) \leq k$ 
for $k \geq 3$. In Section 3, we show that $\lambda_{2,1}^{1,0}(G) \leq \Delta^{2}$ for any claw-free graph $G$ and therefore partially confirm Conjecture 1.2. The paper is concluded with the results on $(1,0)$-relaxed $L(2,1)$-labelings of some infinite families of toroidal grids presented in Section 4 . In particular, we present $(1,0)$-relaxed $L(2,1)$-labeling numbers of three infinite families of toroidal grids and prove that the $(1,0)$-relaxed $L(2,1)$-labeling number of a toroidal grid is bounded from above by 6 .

Given a vertex $v$ in a graph $G$, the set consisting of all neighbors of $v$ is denoted by $N_{G}(v)$ or simply $N(v)$.

Let $[n]$ denote the set $\{0,1, \ldots, n-1\}$. The path $P_{n}$ is the graph whose set of vertices is $[n]$ and in which two vertices are adjacent precisely if their difference is \pm 1 . For an integer $n \geq 3$, the cycle of length $n$ is the graph $C_{n}$ whose vertices are $[n]$ and whose edges are the pairs $\{i, i+1\}$, where the arithmetic is done modulo $n$.

If every vertex in a graph has degree $k$, then we call the graph $k$-regular. In particular, we call a 3-regular graph a cubic graph.

\section{Complexity of $(1,0)$-Relaxed $L(2,1)$-LABeling}

Let $k \geq 3$ be an integer. A $k$-black-and-white coloring of a graph $G$ is a coloring of the vertices of $G$ with labels black and white such that every vertex has exactly two neighbors of the same color and $k-2$ neighbors of the opposite colors. If $G$ admits a $k$-black-and-white coloring, then we can partition the edges of $G$ into three sets: white edges, i.e. edges with two white end-vertices, black edges, i.e. edges with two black end-vertices and black-and-white edges, i.e. edges with one black and one white end-vertex.

Let also a $k$-labeling of a graph $G$ be an assignment of labels from $\{0,1, \ldots, k\}$ to the vertices of $G$.

Let $k \geq 3$ be an integer. The $\mathrm{BW}(k)$-problem is defined as follows.

Problem 2: $\mathrm{BW}(k)$-problem.

Instance: A $k$-regular graph $G$.

Question: Is there a $k$-black-and-white coloring of $G$ ?

It is known that the $\mathrm{BW}(k)$ problem is NP-complete for every $k \geq 3$ [4].

Proposition 2.1. FPRL21 is NP-complete for $k \in\{3,4\}$.

Proof. Let $f$ be a $k$-labeling of a graph $G$. It can be verified in polynomial time whether $f$ is a $(1,0)$-relaxed $k$ - $L(2,1)$-labeling of $G$. It follows that the problem is in the class NP.

For $k=3$ we use a reduction from BW(3). Let $G$ be a 3 -regular graph for which we want to find a 3-blackand-white coloring. We may assume without loss of generality that $G$ is connected. Let $G^{\prime}$ be the graph obtained from $G$ by subdividing each edge of $G$ by two new vertices of degree two.

Claim 1. $G$ admits a BW(3)-coloring if and only if $G^{\prime}$ admits a $(1,0)$-relaxed 3- $L(2,1)$-labeling.

Proof of Claim 1. We first assume that a 3-black-and-white coloring of $G$ is given. Since every white (resp. black) vertex of $G$ has exactly two white (resp. black) neighbors in $G$, the set of white (resp. black) vertices of $G$ induces a 2-regular graph. Note that a 2-regular graph consists of disjoint union of cycles. Moreover, since new vertices of $G^{\prime}$ (i.e. vertices of $V\left(G^{\prime}\right) \backslash V(G)$ ) are also of degree two, the set of white (resp. black) vertices together with the vertices obtained by subdivision of white (resp. black) edges induce a disjoint union of cycles in $G^{\prime}$.

In $G^{\prime}$, we assign label 0 to every white vertex of $G$, and label 3 to every black vertex of $G$. A new vertex on a black-and-white edge adjacent to the white (resp. black ) vertex we label 2 (resp. 1). On each of the cycles that correspond to white vertices we use consecutively the pattern $(0,1,3,0)$. Thus, a vertex labeled 0 , say $v$, has exactly one neighbor labeled 1 , one neighbor labeled 2 and one neighbor labeled 3, i.e. $v$ admits exactly one neighbor with a label one apart while common neighbors of $v$ are assigned different labels. Moreover, none of 2 -neighbors of $v$ is labeled 0 . On each of the cycles that correspond to black vertices we use consecutively the pattern $(3,0,2,3)$. Note that every vertex labeled 3 has exactly one neighbor labeled 0 , one neighbor labeled 1 


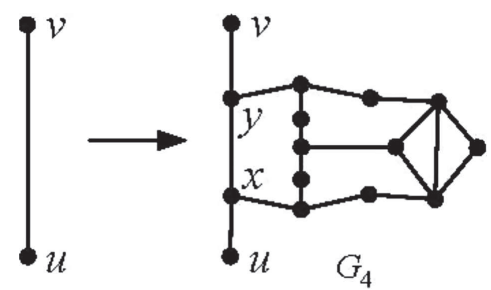

FiguRE 1. Replacement for $k=4$.

and one neighbor labeled 2 . Since all new vertices in $G^{\prime}$ also clearly satisfy the constraints of the $(1,0)$-relaxed $L(2,1)$-labeling, this gives a $(1,0)$-relaxed $3-L(2,1)$-labeling of $G^{\prime}$.

Now assume that $G^{\prime}$ admits a $(1,0)$-relaxed 3- $L(2,1)$-labeling. An original vertex of $G$ has degree three in $G^{\prime}$, so it has to be labeled either 0 (with neighbors labeled $1,2,3$ ) or 3 (with neighbors labeled $0,1,2$ ). It is not difficult to establish that all possible labelings of the path on four vertices with end-vertices labeled 3 or 0 , with respect to the obvious symmetry, are $(0,1,3,0),(0,2,1,3),(0,3,2,0),(3,0,1,3)$, and $(3,0,2,3)$. By the above discussion, an original vertex labeled 0 has exactly one neighbor labeled 1 and an original vertex labeled 3 has exactly one neighbor labeled 2 .

Let $x$ be an original vertex labeled 0 (resp. 3) and $z$ its neighbor in $G$ such that the path obtained by subdividing the edge $x z \in E(G)$ contains the neighbor of $x$ labeled 1 (resp. 2). We will say that that $z$ is the closely linked neighbor of $x$. Note that $z$ is labeled 0 (resp. 3) if $x$ is labeled 0 (resp. 3).

Let us now for every original vertex $x$ and its closely linked neighbor $z$ assign the direction from $x$ towards $z$ to the edge $x z$ in $G$, i.e. $x$ is the tail and $z$ is the head of the directed edge. Since every vertex in $G$ admits exactly one closely linked neighbor, the out-degree of every vertex of $G$ is one. Moreover, since a vertex and its closely linked neighbor are both labeled by the same label, the in-degree of every vertex of $G$ is also one. It follows that the vertices of the path that corresponds to a directed edge with the tail labeled 0 (resp. 3) are labeled with respect to the direction of the edge with the the pattern $(0,1,3,0)$ (resp. $(3,2,0,3))$ in $G^{\prime}$. Moreover, the vertices of the path that corresponds to an undirected edge of $G$ are labeled with the the pattern $(0,2,1,3)$ in $G^{\prime}$. Hence, every vertex of $G$ labeled 0 (resp. 3) admits two neighbors labeled 0 (resp. 3) and one neighbor labeled 3 (resp. 0) in $G$. Since by assigning label black (resp. white) to every vertex labeled 0 (resp. 3 ) in $G$, we clearly obtain a 3 -black-and-white coloring of $G$, Claim 1 is proved.

For $k=4$ we use a reduction from BW(4). Let $G$ be a 4 -regular graph for which we want to find a 4-blackand-white coloring. We show that the graph $G^{\prime}$ obtained from $G$ by replacing each edge of $G$ by the graph $G_{4}$ depicted in Figure 1 admits a $(1,0)$-relaxed 4- $L(2,1)$-labeling if and only if $G$ admits a 4-black-and-white coloring.

Claim 2. $G$ admits a BW(4)-coloring if and only if $G^{\prime}$ admits a $(1,0)$-relaxed $4-L(2,1)$-labeling.

Proof of Claim 2. Note first that vertices $u$ and $v$ of $G_{4}$ are labeled 0 or 4 since both are of degree four in $G^{\prime}$. It can be checked that the vertices of the sequence $u, x, y, v$ allow totally 8 combinations in a possible $(1,0)$ relaxed $4-L(2,1)$-labeling of $G_{4}$. These 8 combinations are listed as follows: $(4,0,2,4),(0,2,4,0),(4,1,3,0)$, $(0,1,3,4),(4,2,0,4),(0,4,2,0),(0,3,1,4),(4,3,1,0)$.

Suppose that $f$ is a $(1,0)$-relaxed $4-L(2,1)$-labeling of $G^{\prime}$. It is already noted that $f(u) \in\{0,4\}$ since $d_{G^{\prime}}(u)=4$. Let $v_{1}, v_{2}, v_{3}, v_{4}$ be the four neighbors of $u$ in $G$, and let $u x_{i} y_{i} v_{i}, i=1,2,3,4$, be the four paths that start with $u$ in $G^{\prime}$. If $u$ is labeled 0 , then $x_{1}, x_{2}, x_{3}, x_{4}$ are labeled with distinct labels from $\{1,2,3,4\}$, say $f\left(x_{i}\right)=i$. Thus, the paths $u x_{i} y_{i} v_{i}, i=1,2,3,4$, are labeled $(0,1,3,4),(0,2,4,0),(0,3,1,4)$ and $(0,4,1,0)$, respectively. If $u$ is labeled 4 , then $x_{1}, x_{2}, x_{3}, x_{4}$ are labeled $0,1,2,3$, say $f\left(x_{i}\right)=i-1$. Hence, the paths $u x_{i} y_{i} v_{i}$, $i=1,2,3,4$ are labeled $(4,0,2,4),(4,1,3,0),(4,2,0,4)$ and $(4,3,1,0)$, respectively. By replacing label 0 with 

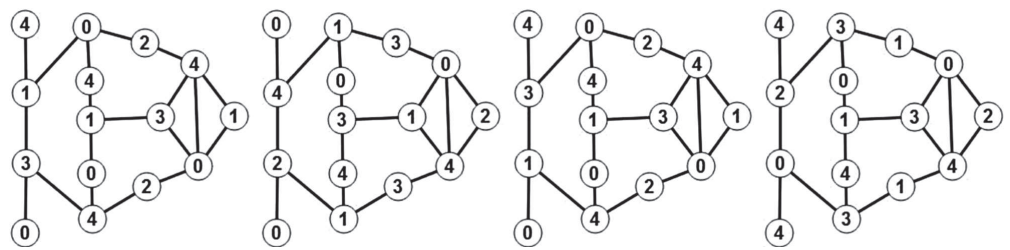

Figure 2. (1,0)-relaxed 4- $L(2,1)$-labelings of $G_{4}$ with $f(u), f(v) \in\{0,4\}$.

color white and label 4 with color black in $G$, we see that the restriction of $f$ to the original vertices of $G$ is a BW(4)-coloring of $G$.

Now assume that $G$ has a BW(4)-coloring. In $G^{\prime}$, we first assign label 0 to every white vertex and label 4 to every black vertex of $G$. Since every white (resp. black) vertex of $G$ is adjacent to exactly two white (resp. black) vertices of $G$, white (resp. black) vertices of $G$ induce a 2-regular graph (i.e. a disjoint union of cycles), hence, the corresponding "white" (resp. "black") cycles in $G^{\prime}$ can be labeled consecutively $(0,2,4,0)$ (resp. $(4,0,2,4)$ ). Analogously, every white (resp. black) vertex of $G$ is adjacent to exactly two black (resp. white) vertices of $G$. It follows that black-and-white edges of $G$ also induce a disjoint union of cycles in $G$. Thus, we can label the paths in $G^{\prime}$ that correspond to black-and-white edges in these cycles consecutively by $(4,1,3,0)$.

Let for $u v \in E(G)$ the 4 -tuple $\left(c_{1}, c_{2}, c_{3}, c_{4}\right)$ denote the labels assigned to vertices $(u, x, y, v)$ in the corresponding $G_{4}$. For each $\left(c_{1}, c_{2}, c_{3}, c_{4}\right)$, one can extend a $(1,0)$-relaxed $4-L(2,1)$-labeling of $G_{4}$ as depicted in Figure 2. Since we obtain a $(1,0)$-relaxed $4-L(2,1)$-labeling of $G^{\prime}$, this case is settled.

Theorem 2.2. FPRL21 is NP-complete for every $k \geq 3$.

Proof. The proof is by induction on $k$. For $k \in\{3,4\}$, the result is obtained in Proposition 2.1.

A star $S_{n}$ is the complete bipartite graph $K_{1, n}$, where the vertex with a degree more that one is called the center of $S_{n}$. We define a double star as the union of two stars with the edge joining the centers. More precisely, for $n \geq m \geq 0$ the double star $S(n, m)$ is the graph with the vertex set $V(S(n, m))=\left\{v_{0}, v_{1}, \ldots, v_{n}, u_{0}, u_{1}, \ldots, u_{m}\right\}$ and the set of edges $E(S(n, m))=\left\{v_{0} u_{0}\right\} \cup\left\{v_{0} v_{i} \mid i=1,2, \ldots, n\right\} \cup\left\{u_{0} u_{j} \mid j=1,2, \ldots, m\right\}$.

Clearly, for $i \in\{1,2, \ldots, n\}$ and $j \in\{1,2, \ldots, m\}, v_{i}$ and $u_{j}$ are vertices of degree 1 in $S(n, m)$. The vertices $v_{0}$ and $u_{0}$ are called the centers of $S(n, m)$, while $v_{i}, i=1,2, \ldots, n$ and $u_{j}, j=1,2, \ldots, m$, are called the leaves of $S(n, m)$.

Let $G$ be a graph for which the existence of $(1,0)$-relaxed $k-L(2,1)$-labeling is to be verified. We will construct a graph $G^{\prime}$ which has a $(1,0)$-relaxed $(k+2)-L(2,1)$-labeling if and only if $G$ admits a $(1,0)$-relaxed $k$ - $L(2,1)$ labeling.

We construct $G^{\prime}$ as follows. For each vertex $x \in V(G)$, we construct a unique double star $S_{x}(k+1, k+1)$, and identify $x$ and an arbitrary leaf of $S_{x}(k+1, k+1)$. That is to say, $x$ and $y$ are adjacent in $G$ if and only if $x$ and $y$ are adjacent in $G^{\prime}$. This completes the construction of $G^{\prime}$. Let $x^{\prime}$ and $x^{\prime \prime}$ denote the centers of $S_{x}(k+1, k+1)$ such that $x^{\prime}$ is adjacent to $x$.

Assume that $G^{\prime}$ admits a $(1,0)$-relaxed $(k+2)-L(2,1)$-labeling. Centers of double stars have degree $k+2$, and therefore each of these centers is labeled either 0 or $k+2$. Note also that two centers in the same double star receive different labels. Moreover, every leaf of $S_{x}(k+1, k+1)$ is adjacent to one center of $S_{x}(k+1, k+1)$ and is at distance two from the other center of $S_{x}(k+1, k+1)$. It follows that leafs cannot be labeled $k+2$ or 0 . Since we showed that $V(G)$ have labels in $\{1, \ldots, k+1\}$, it is trivially to obtain a $(1,0)$-relaxed $k$ - $L(2,1)$-labeling of $G$.

Now we assume that $G$ admits a $(1,0)$-relaxed $k$ - $L(2,1)$-labeling $f$. We construct the labeling $f^{\prime}$ of $G^{\prime}$ as follows. For each $x \in V(G)$ we set $f^{\prime}(x):=f(x)$. Moreover, for each $x \in V(G)$ with $f^{\prime}(x)=k+1$, we label $x^{\prime}$ and $x^{\prime \prime}$ with 0 and $k+2$, respectively, while for each $x \in V(G)$ with $f^{\prime}(x) \neq k+1$ we label $x^{\prime}$ and $x^{\prime \prime}$ with $k+2$ and 0 , 
respectively. Finally, we label vertices of $N_{G^{\prime}}\left(x^{\prime}\right) \backslash\left\{x, x^{\prime \prime}\right\}$ with distinct colors of $\{1,2, \ldots, k+1\} \backslash\left\{f^{\prime}(x)\right\}$ and vertices of $N_{G^{\prime}}\left(x^{\prime \prime}\right) \backslash\left\{x^{\prime}\right\}$ with distinct colors of $\{1,2, \ldots, k+1\}$. One can readily check that $f^{\prime}$ is a $(1,0)$-relaxed $(k+2)-L(2,1)$-labeling of $G^{\prime}$.

\section{Claw-Free GraphS}

A claw is the complete bipartite graph $K_{1,3}$. A claw-free graph is a graph that does not have a claw as an induced subgraph.

In this section, we show that Conjecture 1.2 is true for all claw-free graphs. More formally, we show

Theorem 3.1. If $G$ is a connected claw-free graph, then $\lambda_{2,1}^{1,0}(G) \leq \Delta^{2}$.

The results on $L(2,1)$-labelings of $K_{1, n}$-free graph studied in [10] provide the following

Proposition 3.2. If $G$ is a claw-free graph, then $\lambda(G) \leq \frac{\Delta^{2}}{2}+2 \Delta$.

Since it is straightforward to see that $\lambda_{2,1}^{1,0}(G) \leq \lambda(G)$ for any graph $G$, these two results together with Proposition 3.2 imply that we have to show that Conjecture 1.2 holds also for claw-free graphs with a maximum degree three.

A diamond is a complete graph of order 4 minus one edge.

Lemma 3.3 ([6]). If $G \neq K_{4}$ is a connected, claw-free, cubic graph of order $n$, then the vertex set $V(G)$ can be uniquely partitioned into sets each of which induces a triangle or a diamond in $G$.

By Lemma 3.3, we can obtain a connected, claw-free, cubic graph $G$ from a sequence of vertex-disjoint triangles and diamonds by adding edges between vertices of degree two in the triangles and diamonds. We call every triangle in $G$ a triangle-unit and every diamond a diamond-unit.

Let $G$ be a graph and $S \subseteq V(G)$. Then $G-S$ denotes the subgraph of $G$ induced by $V(G) \backslash S$.

Theorem 3.4. If $G$ is a connected, claw-free graph with $\Delta(G)=3$, then $\lambda_{2,1}^{1,0}(G) \leq 9$.

Proof. If $G=K_{4}$, then $\lambda_{2,1}^{1,0}(G)=4$. In what follows, we assume $G \neq K_{4}$. Suppose the result is false, and let $G$ be a counterexample such that $|V(G)|$ is as small as possible. By the choice of $G, G$ is a connected, claw-free graph of order $n>4$ such that $\lambda_{2,1}^{1,0}(G)>9$, and any connected, claw-free graph $G^{\prime}$ of order $n^{\prime}<n$ has $\lambda_{2,1}^{1,0}\left(G^{\prime}\right) \leq 9$.

If $G$ contain a vertex $u$ of degree one, by the minimality of $G, G-\{u\}$ has a $(1,0)$-relaxed 9- $L(2,1)$ labeling $f^{\prime}$. Since we can easily extend $f$ to a $(1,0)$-relaxed $9-L(2,1)$-labeling of $G$ by properly labeling $u$, we obtain a contradiction.

If $G$ contains a vertex $u$ of degree two, then let $N_{G}(u)=\{v, w\}$ and $G^{\prime}=G-\{u\}$. By the minimality of $G, G^{\prime}$ has a $(1,0)$-relaxed 9-L(2,1)-labeling $f^{\prime}$. If one of $v$ and $w$, say $v$, is of degree less than three in $G$, then let $N_{G^{\prime}}(v)=\left\{v_{1}\right\}$ and $N_{G^{\prime}}(w)=\left\{w_{1}, w_{2}\right\}$. We can label $u$ with an arbitrary label from $\{0,1,2, \ldots, 9\} \backslash\left\{f^{\prime}(v), f^{\prime}(w), f^{\prime}(v)+1, f^{\prime}(v)-1, f^{\prime}(w)+1, f^{\prime}(w)-1, f^{\prime}\left(v_{1}\right), f^{\prime}\left(w_{1}\right), f^{\prime}\left(w_{2}\right)\right\}$ to obtain a (1,0)-relaxed 9-L(2,1)-labeling of $G$, a contradiction. If both $v$ and $w$ are vertices of degree three in $G$, let $N_{G^{\prime}}(v)=\left\{v_{1}, v_{2}\right\}$ and $N_{G^{\prime}}(w)=\left\{w_{1}, w_{2}\right\}$. Consider the set $S=\left\{f^{\prime}(v), f^{\prime}(w), f^{\prime}(v)+1, f^{\prime}(v)-1, f^{\prime}(w)+1, f^{\prime}(w)-1, f^{\prime}\left(v_{1}\right), f^{\prime}\left(v_{2}\right), f^{\prime}\left(w_{1}\right), f^{\prime}\left(w_{2}\right)\right\}$. If $|S| \leq 9$, then we can label $u$ with an arbitrary label from $\{0,1,2, \ldots, 9\} \backslash S$ to obtain a $(1,0)$-relaxed 9- $L(2,1)$-labeling of $G$, a contradiction. If $|S|=10$, then $\left|f^{\prime}\left(v_{i}\right)-f^{\prime}(v)\right| \neq 1,\left|f^{\prime}\left(w_{i}\right)-f^{\prime}(v)\right| \neq 1$, and $\left|f^{\prime}(w)-f^{\prime}(v)\right| \neq 1$. It follows that we can label $u$ with $f^{\prime}(v)+1$ or $f^{\prime}(v)-1$ to obtain a (1,0)-relaxed 9- $L(2,1)$-labeling of $G$, a contradiction.

Since we proved above that $G$ cannot admit vertices of degree one or two, we assume in the following that $G$ is a connected, claw-free, cubic graph of order $n>4$. By Lemma 3.4, $G$ consists of triangle-units and diamond-units.

Claim 1. $G$ contains no diamond-unit. 
Suppose to the contrary that $G$ admits a diamond-unit $D$, where $V(D)=\{a, b, c, d\}, a b \notin E(G)$. Let $N_{G}(a) \backslash\{c, d\}=\{x\}$ and $N_{G}(b) \backslash\{c, d\}=\{y\}$. Define $G^{\prime}$ as the resulting graph obtained from the graph $G-\{a, b, c, d\}$ by connecting $x$ and $y$. Clearly, $G^{\prime}$ is a connected, claw-free, cubic graph. By the minimality of $G$, $G^{\prime}$ has a (1,0)-relaxed 9- $L(2,1)$-labeling $f^{\prime}$. Let $f^{\prime}(x)=c_{1}$ and $f^{\prime}(y)=c_{2}$, where $c_{2}>c_{1}$. Note that $c_{1}, c_{2} \in$ $\{0,1, \ldots, 9\}$. We now extend $f^{\prime}$ to a $(1,0)$-relaxed 9- $L(2,1)$-labeling $f$ of $G$ by labeling $a$ and $b$ with $c_{2}$ and $c_{1}$, respectively, and by labeling $c$ and $d$ with any two labels from $\{0,1, \ldots, 9\} \backslash\left\{c_{1}, c_{2}, c_{1}+1, c_{1}-1, c_{2}+1, c_{2}-1\right\}$. Since we obtain a contradiction, the proof is complete.

Claim 2. $G$ does not contain two triangle-units connected with two or three edges.

Suppose to the contrary that $T_{1}$ and $T_{2}$ are two adjacent triangle-units, where $V\left(T_{1}\right)=\left\{x_{1}, y_{1}, z_{1}\right\}, V\left(T_{2}\right)=$ $\left\{x_{2}, y_{2}, z_{2}\right\}$, and $x_{1} x_{2} \in E(G)$. If $y_{1} y_{2} \in E(G), z_{1} z_{2} \in E(G)$, then it is not difficult to find a (1,0)-relaxed 5 - $L(2,1)$-labeling of $G$ (note that $G$ is isomorphic to the Cartesian product of a triangle and $K_{2}$ ) and we obtain a contradiction. It follows that there is exact one edge between $\left\{y_{1}, z_{1}\right\}$ and $\left\{y_{2}, z_{2}\right\}$. We may assume w.l.o.g. that $y_{1} y_{2} \in E(G)$. Let $N_{G}\left(z_{i}\right) \backslash\left\{x_{i}, y_{i}\right\}=\left\{u_{i}\right\}, i=1,2$ (note that $u_{1} \neq u_{2}$ since $G$ is claw-free). Consider the graph $G^{\prime}$ obtained from $G-\left\{x_{1}, y_{1}, z_{1}, x_{2}, y_{2}, z_{2}\right\}$ by connecting $u_{1}$ and $u_{2}$. Clearly, $G^{\prime}$ is a connected, claw-free, cubic graph. By the minimality of $G, G^{\prime}$ has a $(1,0)$-relaxed 9- $L(2,1)$-labeling $f^{\prime}$. Let $f^{\prime}\left(u_{1}\right)=c_{1}, f^{\prime}\left(u_{2}\right)=c_{2}$, where $c_{2}>c_{1}$. Then, we can extend $f^{\prime}$ to a $(1,0)$-relaxed 9- $L(2,1)$-labeling of $G$ by labeling $z_{1}$ and $z_{2}$ with $c_{2}$ and $c_{1}$, respectively, and by properly labeling $x_{1}, y_{1}$ and $x_{2}, y_{2}$ with the labels from $\{0,1, \ldots, 9\} \backslash\left\{c_{1}, c_{2}, c_{2}+1, c_{2}-1\right\}$ and $\{0,1, \ldots, 9\} \backslash\left\{c_{1}, c_{2}, c_{1}+1, c_{1}-1\right\}$, respectively. Since we obtain a contradiction, and this assertion completes the proof.

From Lemma 3.3 and Claim 1 it follows, that $G$ contains a triangle-unit. Let then $T$ be a triangle-unit induced by the set $\{x, y, z\}$ and let $N_{G}(x) \backslash\{y, z\}=\left\{x_{0}\right\}, N_{G}(y) \backslash\{x, z\}=\left\{y_{0}\right\}, N_{G}(z) \backslash\{x, y\}=\left\{z_{0}\right\}$. By Claim 1, we have $x_{0} \neq y_{0}, x_{0} \neq z_{0}, y_{0} \neq z_{0}$. Let $N_{G}\left(x_{0}\right) \backslash\{x\}=\left\{x_{1}, x_{2}\right\}, N_{G}\left(y_{0}\right) \backslash\{x\}=\left\{y_{1}, y_{2}\right\}$, and $N_{G}\left(z_{0}\right) \backslash\{x\}=\left\{z_{1}, z_{2}\right\}$. Since $G$ is claw-free, $x_{1} x_{2} \in E(G), y_{1} y_{2} \in E(G), z_{1} z_{2} \in E(G)$. By Claim 1 , the sets $\left\{x_{1}, x_{2}\right\},\left\{y_{1}, y_{2}\right\}$, and $\left\{z_{1}, z_{2}\right\}$ have pairwise a non-empty intersection. Moreover, by Claim 2 , we also have $x_{0} \notin\left\{y_{1}, y_{2}, z_{1}, z_{2}\right\}, y_{0} \notin\left\{x_{1}, x_{2}, z_{1}, z_{2}\right\}$, and $z_{0} \notin\left\{x_{1}, x_{2}, y_{1}, y_{2}\right\}$. Let $G^{\prime}:=G-\{x, y, z\}$. Clearly, $G^{\prime}$ is a claw-free graph of maximum degree at most three. By the minimality of $G, G^{\prime}$ admits a $(1,0)$-relaxed 9- $L(2,1)$-labeling $f^{\prime}$. Let $f^{\prime}\left(x_{i}\right)=a_{i}, f^{\prime}\left(y_{i}\right)=b_{i}, f^{\prime}\left(z_{i}\right)=c_{i}$, for $i=0,1,2$.

Let $C_{0}:=\{0,1, \ldots, 9\} \backslash\left\{a_{1}, a_{2}, a_{0}, a_{0}+1, a_{0}-1, b_{0}, c_{0}\right\}, C_{1}:=\{0,1, \ldots, 9\} \backslash\left\{b_{1}, b_{2}, b_{0}, b_{0}+1, b_{0}-1, a_{0}, c_{0}\right\}$, and $C_{2}:=\{0,1, \ldots, 9\} \backslash\left\{c_{1}, c_{2}, c_{0}, c_{0}+1, c_{0}-1, a_{0}, b_{0}\right\}$. Clearly, $\left|C_{i}\right| \geq 3$ for $i=0,1,2$.

If $\left|C_{0} \cup C_{1} \cup C_{2}\right| \geq 4$, then we may assume w.l.o.g. that there exists $a \in C_{0}$ such that $a \notin C_{1}$. Observe that $C_{1}$ contains an element $b$ such that $b \notin\{a-1, a+1, a\}$. Moreover, $C_{2}$ contains an element $c$ such that $c \notin\{a, b\}$. If $|b-a|>2$ or $|b-a|=2$ and $2 c \neq b+a$, we label $x, y$, and $z$ with $a, b$, and $c$, respectively. Since we extend $f^{\prime}$ to a $(1,0)$-relaxed 9- $L(2,1)$-labeling of $G$, we obtain a contradiction. If $|b-a|=2$ and $2 c=b+a$, then we distinguish two cases. If $C_{2} \neq\{a, b, c\}$, we choose the label $c^{\prime} \in C_{2} \backslash\{c, a, b\}$ and label $x, y$, and $z$ with $a, b$, and $c^{\prime}$, respectively. If $C_{2}=\{c, a, b\}$, we select $b^{\prime} \in C_{1} \backslash\{c, b\}$, and label $x, y$, and $z$ with $a, b^{\prime}$, and $b$. Since the obtained labeling is in both cases a $(1,0)$-relaxed 9- $L(2,1)$-labeling of $G$, we come to contradiction.

If $\left|C_{0} \cup C_{1} \cup C_{2}\right|=3$, let $C_{0}=C_{1}=C_{2}:=\{a, b, c\}$. We may assume w.l.o.g that $a<b<c$. If $c \neq a+2$, we label $x, y$ and $z$ with $a, b$, and $c$, respectively to obtain a (1,0)-relaxed 9- $L(2,1)$-labeling of $G$, a contradiction. If $c=a+2$, then $b=a+1$. Note that $\left|C_{0}\right|=3$, which implies that $\left|\left\{a_{1}, a_{2}, a_{0}, a_{0}+1, a_{0}-1, b_{0}, c_{0}\right\}\right|=7$. It follows that $\left|a_{i}-a_{0}\right| \neq 1, i=1,2,\left|b_{0}-a_{0}\right| \neq 1$, and $\left|c_{0}-a_{0}\right| \neq 1$. We now label $x$ with $a_{0}+1$. If $a_{0}+1<a$, label $y$ and $z$ with $b$ and $c$, respectively. If $a_{0}+1>c$, label $y$ and $z$ with $a$ and $b$, respectively. The obtained labeling is a $(1,0)$-relaxed $9-L(2,1)$-labeling of $G$, we come to contradiction. This assertion completes the proof.

Theorems 3.1 and 3.4 together with Proposition 3.2 give useful upper bounds for the $(1,0)$-relaxed $L(2,1)$ labeling number of claw-free graphs. However, the complexity of FPRL21 on this class of graphs remains open. 


\section{TOROIDAL GRIDS}

The Cartesian product of graphs $G$ and $H$ is the graph $G \square H$ with the vertex set $V(G) \times V(H)$ and $\left(x_{1}, x_{2}\right)\left(y_{1}, y_{2}\right) \in E(G \square H)$ whenever $x_{1} y_{1} \in E(G)$ and $x_{2}=y_{2}$, or $x_{2} y_{2} \in E(H)$ and $x_{1}=y_{1}$. Let $u \in V(G)$.

The subgraph of $G \square H$ induced by $\{u\} \times V(H)$ is isomorphic to $H$. It is called an $H$-fiber and is denoted by $H^{u}$. In particular, the subgraph of $C_{m} \square C_{n}$ induced by a vertex $i$ of $C_{m}$ is denoted by $C_{n}^{i}$.

The Cartesian product of two cycles $C_{m} \square C_{n}$ is also called a toroidal grid and denoted by $T_{m, n}$. Note that $L(2,1)$-labelings of toroidal grids are considered in [9].

Note that $T_{m, n}$ is a 4-regular graph. Suppose that $T_{m, n}$ admits a $(1,0)$-relaxed $4-L(2,1)$-labeling $f$. If $u$ is a vertex of $T_{m, n}$, then $f(u)$ is either 0 or 4 . Since all neighbors of $u$ cannot be labeled 0 or 4 , we have the following general lower bound.

Proposition 4.1. $\lambda_{2,1}^{1,0}\left(T_{m, n}\right) \geq 5$.

In the following, we will use a rectangular pattern with $m$ rows and $n$ columns to represent a labeling of $T_{m, n}$ in a natural way. If $P$ and $Q$ are rectangular patterns which represent a labeling of $T_{m, n}$ and $T_{m, \ell}$, respectively, then $P Q$ denotes the rectangular pattern with $m$ rows and $n+\ell$ columns obtained by concatenating $P$ and $Q$, such that $P Q$ represents a labeling of $T_{m, n+\ell}$. Moreover, $P^{k}$ represents a labeling of $T_{m, k n}$ made by the rectangular pattern with $m$ rows and $k n$ columns obtained by concatenating $k$ copies of $P$.

As an example, let

$$
P=\left[\begin{array}{llll}
1 & 4 & 3 & 0 \\
5 & 2 & 1 & 4 \\
3 & 0 & 5 & 2
\end{array}\right] \text { and } Q=\left[\begin{array}{cc}
5 & 2 \\
3 & 0 \\
1 & 4
\end{array}\right]
$$

Obviously, $P^{k}$ represents a $(1,0)$-relaxed 5 - $L(2,1)$-labeling of $T_{3,4 k}$, while $P^{k} Q$ represents a $(1,0)$-relaxed 5 - $L(2,1)$-labeling of $T_{3,4 k+2}$.

The patterns that provide upper bounds for the $(1,0)$-relaxed $L(2,1)$-labeling number of $T_{m, n}$ which are not present in the proofs of the following theorems are given in the Appendices A to C.

Theorem 4.2. Let $n \geq 3$. Then

$$
\lambda_{2,1}^{1,0}\left(T_{3, n}\right)= \begin{cases}8, & n=3 \\ 7, & n=5, \\ 6, & n \geq 7, \quad n \text { is odd } \\ 5, & n \text { is even }\end{cases}
$$

Proof. We show above that $P^{k}$ and $P^{k} Q$ represents a (1,0)-relaxed 5- $L(2,1)$-labeling of $T_{3,4 k}$ and a $(1,0)$-relaxed 5 - $L(2,1)$-labeling of $T_{3,4 k+2}$, respectively. It follows that $\lambda_{2,1}^{1,0}\left(T_{3, n}\right) \leq 5$ for every even $n$. Thus, the upper bound on $\lambda_{2,1}^{1,0}\left(T_{3, n}\right)$ is proved for every even $n$.

Analogously, we found two patterns which obtain a (1,0)-relaxed 6 - $L(2,1)$-labeling of $T_{3,4 k+5}$ and $(1,0)$ relaxed 6 - $L(2,1)$-labeling of $T_{3,4 k+7}$, for any $k \geq 1$. Thus, the upper bound on $\lambda_{2,1}^{1,0}\left(T_{3, n}\right)$ is established also for every odd $n \geq 5$.

Since we also found a $(1,0)$-relaxed 8 - $L(2,1)$-labeling of $T_{3,3}$, a $(1,0)$-relaxed 7 - $L(2,1)$-labeling of $T_{3,5}$, and a $(1,0)$-relaxed $6-L(2,1)$-labeling of $T_{3,7}$, upper bounds are confirmed for every $n$.

Note that the lower bound for even $n$ follows from Proposition 4.1. In order to prove the lower bound for odd $n$, suppose that $f$ is a $(1,0)$-relaxed 5 - $L(2,1)$-labeling of $T_{3, n}$. Let $C_{3}^{i}, i \in[n]$, denote a $C_{3}$ fiber in $T_{3, n}$ and let $F_{i}$ be the set of labels assigned by $f$ to the vertices $C_{3}^{i}$. Consider now the vertices of two consecutive $C_{3}$ fibers $C_{3}^{i}$ and $C_{3}^{i+1}, i \in[n]$ (the arithmetic is done modulo $n$ ). Note that the vertices of $C_{3}^{i}$ and $C_{3}^{i+1}$ induce a graph isomorphic to $C_{3} \square K_{2}$. We can see that that any two vertices of $V\left(C_{3}^{i}\right) \cup V\left(C_{3}^{i+1}\right)$ are at distance at most two in $T_{3, n}$. Thus, $\left|F_{i}\right|+\left|F_{i+1}\right|=6$, i.e. $f$ assigns six different labels to the vertices of $V\left(C_{3}^{i}\right) \cup V\left(C_{3}^{i+1}\right)$. Moreover, we have $F_{i}=F_{i+2}$ for every $i \in[n]$. It follows that $n$ must be even if $\lambda_{2,1}^{1,0}\left(T_{3, n}\right)=5$. 
Suppose now that $f$ is a $(1,0)$-relaxed 6 - $L(2,1)$-labeling of $T_{3,5}$ and let $F_{i}$ be the set of labels assigned by $f$ to the vertices $C_{3}^{i}, i \in[5]$. From the discussion above it follows that $\left|F_{i}\right|+\left|F_{i+1}\right|=6$. Moreover, we can see that $\left|F_{i} \cap F_{i+2}\right| \geq 2$. In particular, $\left|F_{3} \cap F_{0}\right| \geq 2$ and $\left|F_{3} \cap F_{1}\right| \geq 2$. Since $\left|F_{0} \cap F_{1}\right|=0$, we have $\left|F_{3}\right| \geq 4$ and we obtain a contradiction. Thus, $\lambda_{2,1}^{1,0}\left(T_{3,5}\right) \geq 7$.

If $u$ is a vertex of $V\left(T_{3,3}\right)$, then every vertex of $V\left(T_{3,3}\right) \backslash\{u\}$ is at distance at most two from $u$. It follows that we need nine labels for a $(1,0)$-relaxed $L(2,1)$-labeling of $T_{3,3}$. The assertion completes the proof.

Theorem 4.3. Let $n \geq 4$. Then

$$
\lambda_{2,1}^{1,0}\left(T_{4, n}\right)=\left\{\begin{array}{lll}
5, & n \geq 5, & n \equiv 0(\bmod 3) \\
6, & n \geq 5, & n \neq \equiv(\bmod 3) \\
7, & n=4 . &
\end{array}\right.
$$

Proof. Most upper bounds follow from a $(1,0)$-relaxed 5 - $L(2,1)$-labeling of $T_{4,3}$ which together with a 6 -labeling of $T_{4,5}$ and 6-labeling of $T_{4,7}$ provide a $(1,0)$-relaxed 5 - $L(2,1)$-labeling of $T_{4,3 k}$ as well as a $(1,0)$-relaxed 6 - $L(2,1)$ labelings of $T_{4,3 k+5}$ and $T_{4,3 k+7}$. Since a $(1,0)$-relaxed $7-L(2,1)$-labeling of $T_{4,4}$, a $(1,0)$-relaxed 6 - $L(2,1)$-labeling of $T_{4,5}$ and a $(1,0)$-relaxed 6 - $L(2,1)$-labeling of $T_{4,7}$ give the desired upper bounds for $n=4,5,7$, all upper bounds are settled.

By Proposition 4.1 we have $\lambda_{2,1}^{1,0}\left(T_{4, n}\right) \geq 5$. Since we established by a computer that $\lambda_{2,1}^{1,0}\left(T_{4,4}\right) \geq 7$, we are left to show that $\lambda_{2,1}^{1,0}\left(T_{4, n}\right) \geq 6$ for $n \not \equiv 0(\bmod 3)$.

All additions are modulo 4 in the sequel. Let $f$ be a $(1,0)$-relaxed 5 - $L(2,1)$-labeling of $T_{4, n}$ and let $f(j, i)$ denote the assignment of $f$ to a vertex $(j, i) \in T_{4, n}, j \in[4], i \in[n]$. Let also $F_{i}$ denote the set of labels assigned by $f$ to the vertices of $C_{4}^{i}$. Clearly, $\left|F_{i}\right|=4$. Note also that if $f(j, i)=f(k, i+1)$, then $k=j+2$.

Since $\left|F_{i}\right|=4$ and $\left|F_{i} \cup F_{i+1}\right| \leq 6$, we have $\left|F_{i} \cap F_{i+1}\right| \geq 2$. We first show that $\left|F_{i} \cap F_{i+1}\right|=2$. Suppose to the contrary that $\left|F_{i} \cap F_{i+1}\right| \geq 3$. We can assume w.l.o.g. $f(0, i)=f(2, i+1), f(1, i)=f(3, i+1)$ and $f(2, i)=$ $f(0, i+1)$. Since $f$ is a 5 -labeling and $\left|F_{i} \cap F_{i+1}\right| \geq 3$, we have $\left|[6] \backslash\left(F_{i} \cap F_{i+1}\right)\right| \leq 3$. It follows that there exists at least one $j$ such that $f(j, i+2) \in\{f(0, i), f(1, i), f(2, i)\}$. But then we have $f(j, i+2)=f(j+2, i+1)=f(j, i)$ and we obtain a contradiction.

Since $\left|F_{i} \cap F_{i+1}\right|=2$ and $\left|F_{i} \cup F_{i+1}\right|=6$, we may partition $F_{i}$ into two sets $P_{i}$ and $N_{i}$, such that $N_{i}=F_{i} \cap F_{i+1}=P_{i+1}$. Note that

$$
P_{i}+N_{i}+N_{i+1}=[6]
$$

and

$$
P_{i}+P_{i+1}+N_{i+1}=[6] .
$$

By (4.2), we also have $P_{i+1}+P_{i+2}+N_{i+2}=$ [6]. Since $P_{i+1}=N_{i}$ and $P_{i+2}=N_{i+1}$, we have $N_{i}+N_{i+1}+N_{i+2}=[6]$. From (4.1) then it follows that $N_{i+2}=P_{i}$. Since $N_{i+3}=P_{i+1}=N_{i}$ and $P_{i+3}=N_{i+2}=P_{i}$, we have $F_{i}=F_{i+3}$. It is straightforward to see now that $f$ exists only if $n \equiv 0(\bmod 3)$.

Theorem 4.4. If $n \geq 5$, then $\lambda_{2,1}^{1,0}\left(T_{5, n}\right)=6$.

Proof. A (1,0)-relaxed 6- $L(2,1)$-labeling of $T_{5,4}$ together with 6-labelings of $T_{5,5}, T_{5,6}$ and $T_{5,6}$ provide a $(1,0)$ relaxed $6-L(2,1)$-labeling of $T_{5,4 k}$ and a $(1,0)$-relaxed $6-L(2,1)$-labeling of $T_{5,4 k+i}, i \in\{5,6,7\}$. Moreover, since (1,0)-relaxed 6 - $L(2,1)$-labelings of $T_{5,5}, T_{5,6}$, and $T_{5,7}$, give the desired labelings for $n \in\{5,6,7\}$, the upper bound is settled.

In order to establish the lower bound, let assume that all additions are modulo 5 in the sequel.

Suppose to the contrary that there exists a $(1,0)$-relaxed 5 - $L(2,1)$-labeling $f$ of $T_{5, n}$. Let $f(j, i)$ denote the assignment of $f$ to a vertex $(j, i) \in T_{5, n}, j \in[5], i \in[n]$. Let also $F_{i}$ denote the set of labels assigned by $f$ to the vertices of $C_{5}^{i}$. Clearly, $\left|F_{i}\right|=5$. Moreover,

Claim 1. If $f(j, i)=f(k, i \pm 1)$, then either $k=j+2$ or $k=j+3$. 
If $f(j, i)=c$, such that $c-1 \in F_{i}$ and $c+1 \in F_{i}$, then we say that the vertex $(j, i) \in F_{i}$ is squeezed.

Claim 2. Let $c^{\prime}$ be a label of $f$ such that $c^{\prime} \notin F_{i}$. If $f(j, i)=c$ and $(j, i)$ is squeezed, then either $f(j, i+1)=c^{\prime}$ or $f(j, i-1)=c^{\prime}$.

Proof of Claim 2. Let $f(j, i)=c$. Since $(j, i)$ is squeezed, $c-1 \in F_{i}$ and $c+1 \in F_{i}$. Suppose to the contrary that $f(j, i+1), f(j, i-1) \in F_{i}$. Note that $f(j+1, i)$ and $f(j-1, i)$ cannot be both from $\{c-1, c+1\}$. Suppose first that neither of $f(j+1, i)$ and $f(j-1, i)$ is from $\{c-1, c+1\}$. It follows that $f(j+2, i)$ and $f(j-2, i)$ are both from $\{c-1, c+1\}$. If $f(j, i \pm 1) \in F_{i}$, then, by Claim $1, f(j, i \pm 1) \in\{c-1, c+1\}$. Since $(i, j)$ cannot admit two two neighbors with labels from the set $\{f(j, i)-1, f(j, i)+1\}$, we obtain a contradiction. Suppose now w.l.o.g. that $f(j+1, i)=c-1$. Suppose also w.l.o.g. that $f(j+2, i)=c+1$. If $f(j, i+1), f(j, i-1) \in F_{i}$, then, by Claim $1, f(j, i+1), f(j, i-1) \in\{c+1, f(j-2, i)\}$. Since $(j, i)$ has already a neighbor labeled $c-1$, we have $f(j, i+1) \neq c-1$ and $f(j, i-1) \neq c-1$. But then $f(j, i+1)=f(j, i-1)=f(j-2, i)$ and we obtain a contradiction.

We now consider the following three cases.

Case 1. $F_{i}=\{0,1,2,3,4\}$.

Since vertices labeled 2,3 , and 4 are squeezed in $F_{i}$, by Claim 2 , we need to label 5 three vertices in $V\left(C_{5}^{i-1}\right) \cup V\left(C_{5}^{i+1}\right)$. But then either $\left|F_{i-1}\right|<5$ or $\left|F_{i+1}\right|<5$ and we obtain a contradiction. Thus, $F_{i} \neq\{0,1,2,3,4\}$.

Case 2. $F_{i}=\{0,2,3,4,5\}$.

We first show that for $f(j, i)=3$ we have $f(j \pm 1, i) \neq 4$. Suppose to the contrary that $f(j, i)=3$ and $f(j+1, i)=4$. From Case 1 it follows that $F_{i \pm 1} \neq\{0,1,2,3,4\}$. Therefore, $5 \in F_{i \pm 1}$. Since $f(j+2, i) \neq 5$, from Claim 1 it follows that either $f(j+1, i+1)=5$ or $f(j+1, i-1)=5$. But then $(j+1, i)$ admits two neighbors with labels from the set $\{f(j+1, i)-1, f(j+1, i)+1\}$ and we obtain a contradiction.

Since we showed that $C_{5}^{i}$ cannot admit consecutive vertices labeled 3 and 4 , we are left with the following six labelings of $((j, i),(j+1, i), \ldots,(j+4, i)):(3,0,4,2,5),(3,0,4,5,2),(3,2,4,0,5),(3,2,4,5,0),(3,5,4,0,2)$, and $(3,5,4,2,0)$.

Suppose that $((j, i),(j+1, i), \ldots,(j+4, i))=(3,0,4,2,5)$. Vertices labeled 3 and 4 are squeezed in $F_{i}$. Hence, by Claim 2, we need to label 1 one vertex in $V\left(C_{5}^{i-1}\right)$ and $V\left(C_{5}^{i+1}\right)$, respectively. Since $5 \in F_{i \pm 1}$, we have either $f(j+2, i+1)=5$ or $f(j+2, i-1)=5$. Suppose w.l.o.g. that $f(j+2, i+1)=5$. It follows that $f(j+2, i-1)=1$ and $f(j, i+1)=1$. By Claim 1 , we then get $f(j+1, i+1)=2$ and $f(j+1, i+1)=0$. But then $(j, i)$ admits two neighbors with labels from the set $\{f(j, i)-1, f(j, i)+1\}$ and we obtain a contradiction. The proof for the other five labelings is analogous. Thus, $F_{i} \neq\{0,2,3,4,5\}$.

Note that because of the obvious symmetry, Cases 1 and 2 also yield $F_{i} \neq\{1,2,3,4,5\}$ and $F_{i} \neq\{0,1,2,3,5\}$, respectively.

Case 3. $F_{i}=\{0,1,2,4,5\}$.

We first show that for $f(j, i)=1$ we have $f(j \pm 1, i) \neq 2$. Suppose to the contrary that $f(j, i)=1$ and $f(j+1, i)=2$. From Case 1 it follows that $F_{i \pm 1} \neq\{1,2,3,4,5\}$. Therefore, $0 \in F_{i \pm 1}$. Since $f(j-1, i) \neq 0$, from Claim 1 it follows that either $f(j, i+1)=0$ or $f(j, i-1)=0$. But then $(j, i)$ admits two neighbors with labels from the set $\{f(j, i)-1, f(j, i)+1\}$ and we obtain a contradiction.

Since we showed that $C_{5}^{i}$ cannot admit consecutive vertices labeled 1 and 2, we are left with the following six labelings of $((j, i),(j+1, i), \ldots,(j+4, i)):(1,0,2,4,5),(1,0,2,5,4),(1,4,2,0,5),(1,4,2,5,0),(1,5,2,0,4)$, and $(1,5,2,4,0)$.

Suppose that $((j, i),(j+1, i), \ldots,(j+4, i))=(1,0,2,4,5)$. Vertex labeled 1 is squeezed and has a neighbor labeled 0 in $F_{i}$. Hence, by Claim 2 , we need to label 3 either $(j, i-1)$ or $(j, i+1)$. Suppose w.l.o.g. that $f(j, i+1)=3$. By Claim 1, we then get $f(j+1, i+1)=4$ and $f(j+2, i+1)=5$. But then $(j+1, i+1)$ admits two neighbors with labels from the set $\{f(j+1, i+1)-1, f(j+1, i+1)+1\}$ and we obtain a contradiction. The proof for the other five labelings is analogous. Thus, $F_{i} \neq\{0,1,2,4,5\}$. Moreover, because of the obvious symmetry, we also have $F_{i} \neq\{0,1,3,4,5\}$. 
Since we prove that $f$ cannot exist, the proof is complete.

Given two integers $r$ and $s$, let $S(r, s)$ denote the set of all nonnegative integer combinations of $r$ and $s$ :

$$
S(r, s)=\left\{\alpha r+\beta s: \alpha, \beta \in Z^{+}\right\} .
$$

We need the following result of Sylvester.

Lemma 4.5 ([12]). If $r, s>1$ are relatively prime nonnegative integers, then $t \in S(r, s)$ for all $t \geq(s-1)(r-1)$.

Theorem 4.6. If $m, n \geq 64$, then $\lambda_{2,1}^{1,0}\left(T_{m, n}\right) \leq 6$.

Proof. Let

$$
\begin{aligned}
& A=\left[\begin{array}{lllll}
3 & 6 & 5 & 1 & 0 \\
1 & 0 & 3 & 6 & 4 \\
5 & 2 & 4 & 0 & 3 \\
0 & 3 & 6 & 5 & 1 \\
4 & 1 & 0 & 3 & 6
\end{array}\right], \quad B=\left[\begin{array}{llllllllllll}
3 & 5 & 2 & 6 & 0 & 3 & 5 & 1 & 2 & 4 & 6 & 0 \\
1 & 0 & 3 & 1 & 4 & 2 & 6 & 4 & 5 & 1 & 2 & 4 \\
6 & 2 & 5 & 0 & 3 & 5 & 1 & 0 & 3 & 6 & 0 & 3 \\
0 & 3 & 6 & 4 & 1 & 0 & 4 & 5 & 1 & 2 & 5 & 6 \\
4 & 1 & 0 & 3 & 5 & 6 & 2 & 3 & 6 & 0 & 3 & 2
\end{array}\right], \\
& C=\left[\begin{array}{lllll}
3 & 6 & 4 & 2 & 5 \\
1 & 5 & 3 & 6 & 0 \\
4 & 2 & 0 & 1 & 3 \\
0 & 3 & 5 & 4 & 6 \\
1 & 6 & 2 & 0 & 5 \\
4 & 0 & 3 & 6 & 2 \\
3 & 5 & 1 & 4 & 0 \\
1 & 4 & 0 & 2 & 6 \\
5 & 2 & 6 & 1 & 3 \\
6 & 1 & 3 & 5 & 2 \\
0 & 5 & 2 & 6 & 4 \\
2 & 4 & 0 & 3 & 5
\end{array}\right], \quad D=\left[\begin{array}{llllllllllll}
3 & 6 & 5 & 1 & 2 & 4 & 5 & 0 & 2 & 4 & 6 & 5 \\
1 & 4 & 3 & 6 & 0 & 1 & 3 & 6 & 1 & 5 & 2 & 0 \\
5 & 2 & 0 & 4 & 3 & 5 & 0 & 2 & 4 & 0 & 3 & 6 \\
0 & 3 & 5 & 1 & 6 & 4 & 1 & 5 & 6 & 2 & 1 & 5 \\
1 & 6 & 2 & 0 & 5 & 2 & 6 & 3 & 1 & 4 & 6 & 3 \\
4 & 0 & 3 & 6 & 1 & 3 & 5 & 0 & 2 & 5 & 0 & 2 \\
3 & 5 & 1 & 2 & 4 & 6 & 1 & 4 & 6 & 3 & 4 & 6 \\
1 & 2 & 4 & 0 & 3 & 5 & 0 & 2 & 5 & 1 & 2 & 0 \\
5 & 0 & 6 & 5 & 1 & 2 & 4 & 6 & 0 & 4 & 6 & 3 \\
6 & 4 & 2 & 3 & 6 & 0 & 3 & 5 & 1 & 3 & 0 & 2 \\
0 & 3 & 5 & 1 & 2 & 4 & 6 & 2 & 4 & 5 & 1 & 4 \\
2 & 6 & 0 & 4 & 5 & 1 & 0 & 3 & 6 & 0 & 3 & 5
\end{array}\right] .
\end{aligned}
$$

Let also $P_{1}=A^{p} B$ and $P_{2}=C^{p} D$ for $p \geq 1$. We now construct

$$
Q=\left[\begin{array}{c}
P_{1} \\
\vdots \\
P_{1} \\
P_{2}
\end{array}\right], \quad \text { where } P_{1} \text { is repeated } q \text { times and } q \geq 1
$$

Obviously, $Q$ denotes the pattern with $5 q+12$ rows and $5 p+12$ columns.

Since the patterns $A$ and $P_{1}$ constitute a $(1,0)$-relaxed $6-L(2,1)$-labeling of $T_{5,5}$ and $T_{5,5 p+12}$, respectively, while the leftmost five columns of $Q$ constitute a $(1,0)$-relaxed $6-L(2,1)$-labeling of $T_{5 q+12,5}$, it follows that $T_{5 p+17 \alpha, 5 q+17 \beta}$ admits a $(1,0)$-relaxed 6 - $L(2,1)$-labeling for any $p, q, \alpha, \beta \geq 1$. By Lemma 4.5 , we have $\lambda_{2,1}^{1,0}\left(T_{m, n}\right) \leq 6$ for $m, n \geq 64$.

Theorem 4.6 shows that the $(1,0)$-relaxed $L(2,1)$-labeling number of $T_{n, m}$ is bounded above by six whenever $n$ and $m$ are sufficiently large. Note that Theorems 4.2-4.4 imply that $\lambda_{2,1}^{1,0}\left(T_{m, n}\right) \leq 6$ if $m \geq 5$ and $n$ is multiple of three, four or five. Moreover, we have confirmed by a computer search, that $(1,0)$-relaxed $L(2,1)$-labeling number of $T_{n, m}$ is at most six for various other values of $n$ and $m$. Therefore, we propose the following conjecture:

Conjecture 4.7. If $m, n \geq 5$, then $\lambda_{2,1}^{1,0}\left(T_{m, n}\right) \leq 6$. 


\section{Appendix A. Patterns for toroidal grids $T_{3, n}$}

Let

$$
P=\left[\begin{array}{llll}
1 & 4 & 3 & 0 \\
5 & 2 & 1 & 4 \\
3 & 0 & 5 & 2
\end{array}\right] \text { and } Q=\left[\begin{array}{ll}
5 & 2 \\
3 & 0 \\
1 & 4
\end{array}\right]
$$

$P^{k}$ represents a $(1,0)$-relaxed 5 - $L(2,1)$-labeling of $T_{3,4 k}$, while $P^{k} Q$ represents a $(1,0)$-relaxed 5 - $L(2,1)$-labeling of $T_{3,4 k+2}$.

Let

$$
\begin{aligned}
R & =\left[\begin{array}{lllllllll}
6 & 0 & 5 & 3 & 1 & 4 & 5 & 1 & 3 \\
2 & 4 & 1 & 0 & 5 & 6 & 2 & 4 & 0 \\
1 & 3 & 6 & 4 & 2 & 0 & 3 & 6 & 5
\end{array}\right] \text { and } \\
S & =\left[\begin{array}{lllllllllll}
6 & 0 & 4 & 3 & 6 & 5 & 1 & 0 & 4 & 2 & 3 \\
2 & 3 & 1 & 5 & 4 & 0 & 6 & 3 & 1 & 6 & 5 \\
4 & 5 & 2 & 0 & 1 & 3 & 4 & 2 & 5 & 0 & 1
\end{array}\right]
\end{aligned}
$$

By repeating the leftmost four columns of $R$ and $S$, we obtain a (1,0)-relaxed 6- $L(2,1)$-labeling of $T_{3,4 k+5}$ and $T_{3,4 k+7}$, respectively, for any $k \geq 1$.

Let

$$
P_{3}=\left[\begin{array}{lll}
5 & 8 & 4 \\
3 & 6 & 2 \\
7 & 1 & 0
\end{array}\right], \quad P_{5}=\left[\begin{array}{lllll}
2 & 0 & 6 & 5 & 3 \\
4 & 5 & 1 & 7 & 6 \\
7 & 3 & 4 & 0 & 1
\end{array}\right], \quad \text { and } \quad P_{7}=\left[\begin{array}{ccccccc}
2 & 0 & 6 & 1 & 4 & 3 & 5 \\
3 & 1 & 4 & 5 & 2 & 6 & 0 \\
6 & 5 & 2 & 3 & 0 & 1 & 4
\end{array}\right]
$$

$P_{3}, P_{5}$, and $P_{7}$ represent a $(1,0)$-relaxed 8- $L(2,1)$-labeling of $T_{3,3}$, a $(1,0)$-relaxed $7-L(2,1)$-labeling of $T_{3,5}$, and a $(1,0)$-relaxed 6 - $L(2,1)$-labeling of $T_{3,7}$, respectively.

\section{Appendix B. Patterns for toroidal GRIDS $T_{4, n}$}

Let

$$
\begin{aligned}
P & =\left[\begin{array}{lll}
4 & 0 & 2 \\
1 & 3 & 5 \\
0 & 2 & 4 \\
3 & 5 & 1
\end{array}\right], Q_{5}=\left[\begin{array}{lllll}
4 & 0 & 3 & 6 & 2 \\
1 & 6 & 4 & 0 & 5 \\
0 & 3 & 1 & 2 & 6 \\
6 & 2 & 5 & 4 & 1
\end{array}\right], \quad Q_{7}=\left[\begin{array}{lllllll}
4 & 1 & 2 & 4 & 3 & 6 & 2 \\
0 & 3 & 5 & 6 & 1 & 0 & 5 \\
6 & 2 & 0 & 3 & 4 & 2 & 6 \\
3 & 5 & 6 & 1 & 0 & 5 & 1
\end{array}\right], \\
P_{4} & =\left[\begin{array}{llll}
7 & 2 & 3 & 5 \\
0 & 5 & 6 & 4 \\
3 & 1 & 2 & 7 \\
6 & 4 & 0 & 1
\end{array}\right], \quad P_{5}=\left[\begin{array}{lllll}
6 & 2 & 1 & 4 & 0 \\
1 & 5 & 3 & 6 & 2 \\
3 & 6 & 2 & 0 & 5 \\
4 & 0 & 5 & 3 & 1
\end{array}\right], \quad \text { and } P_{7}=\left[\begin{array}{rrrrrrr}
1 & 4 & 6 & 2 & 0 & 4 & 3 \\
2 & 0 & 5 & 3 & 1 & 6 & 5 \\
4 & 6 & 2 & 0 & 5 & 3 & 1 \\
5 & 3 & 1 & 4 & 6 & 2 & 0
\end{array}\right] .
\end{aligned}
$$

For any $k \geq 1$ and $i \in\{5,7\}$, the patterns $P^{k}$ and $P^{k} Q_{i}$ represent a (1,0)-relaxed 5- $L(2,1)$-labeling of $T_{4,3 k}$ and a $(1,0)$-relaxed $6-L(2,1)$-labeling of $T_{4,3 k+i}$, respectively. The patterns $P_{4}, P_{5}$ and $P_{7}$ give a $(1,0)$-relaxed 7- $L(2,1)$-labeling of $T_{4,4}$, a $(1,0)$-relaxed 6 - $L(2,1)$-labeling of $T_{4,5}$ and a $(1,0)$-relaxed 6 - $L(2,1)$-labeling of $T_{4,7}$, respectively. 


\section{Appendix C. Patterns For toroidal GRIDs $T_{5, n}$}

Let

$$
\begin{aligned}
& P=\left[\begin{array}{llll}
5 & 0 & 1 & 3 \\
1 & 2 & 4 & 6 \\
3 & 5 & 0 & 2 \\
6 & 1 & 3 & 5 \\
2 & 4 & 6 & 0
\end{array}\right], \quad P_{5}=\left[\begin{array}{lllll}
4 & 6 & 0 & 3 & 5 \\
0 & 3 & 2 & 4 & 1 \\
5 & 1 & 6 & 0 & 3 \\
6 & 0 & 3 & 2 & 4 \\
2 & 5 & 1 & 6 & 0
\end{array}\right], \quad Q_{5}=\left[\begin{array}{lllll}
4 & 6 & 2 & 4 & 3 \\
0 & 5 & 3 & 0 & 6 \\
3 & 1 & 6 & 5 & 2 \\
6 & 4 & 0 & 3 & 4 \\
1 & 3 & 5 & 1 & 0
\end{array}\right] \\
& Q_{6}=\left[\begin{array}{llllll}
5 & 4 & 0 & 2 & 4 & 3 \\
1 & 2 & 5 & 3 & 0 & 6 \\
4 & 0 & 1 & 6 & 5 & 2 \\
6 & 3 & 4 & 0 & 3 & 4 \\
2 & 1 & 6 & 5 & 1 & 0
\end{array}\right], \quad P_{6}=\left[\begin{array}{llllll}
6 & 1 & 3 & 2 & 0 & 5 \\
0 & 4 & 6 & 5 & 3 & 1 \\
5 & 3 & 1 & 0 & 2 & 6 \\
1 & 6 & 5 & 3 & 4 & 0 \\
4 & 2 & 0 & 6 & 1 & 3
\end{array}\right] \\
& Q_{7}=\left[\begin{array}{lllllll}
5 & 1 & 3 & 6 & 2 & 0 & 4 \\
0 & 2 & 4 & 1 & 5 & 3 & 6 \\
3 & 5 & 0 & 3 & 6 & 4 & 0 \\
1 & 6 & 2 & 4 & 1 & 2 & 5 \\
2 & 4 & 5 & 0 & 3 & 6 & 1
\end{array}\right], \quad \text { and } \quad P_{7}=\left[\begin{array}{lllllll}
1 & 6 & 5 & 3 & 2 & 5 & 3 \\
0 & 4 & 2 & 0 & 6 & 4 & 2 \\
3 & 1 & 6 & 4 & 1 & 0 & 5 \\
2 & 5 & 0 & 2 & 5 & 3 & 6 \\
4 & 3 & 1 & 6 & 4 & 1 & 0
\end{array}\right]
\end{aligned}
$$

For any $k \geq 1$ and $i \in\{5,6,7\}$, the patterns $P^{k}$ and $P^{k} Q_{i}$ constitute a $(1,0)$-relaxed 6- $L(2,1)$-labeling of $T_{5,4 k}$ and $T_{5,4 k+i}$, respectively. The patterns $P_{5}, P_{6}$ and $P_{7}$ give a $(1,0)$-relaxed $6-L(2,1)$-labeling of $T_{5,5}$, a (1,0)-relaxed 6- $L(2,1)$-labeling of $T_{5,6}$ and a $(1,0)$-relaxed 6 - $L(2,1)$-labeling of $T_{5,7}$, respectively.

Acknowledgements. The authors acknowledge the financial support from the National Key R\&D Program of China (grant no. 2019YFA0706402), the Natural Science Foundation of Guangdong Province (grant no. 2018A0303130115), the Slovenian Research Agency (research core funding no. P1-0297, projects J1-1693 and J1-9109), and the National Natural Science Foundation of China (grant nos. 61672051, 61309015, 61702075).

\section{REFERENCES}

[1] B. Dai and W. Lin, On $(s, t)$-relaxed L(2,1)-labelings of the square lattice. Inf. Process. Lett. 113 (2013) $704-709$.

[2] B. Dai and W. Lin, On $(s, t)$-relaxed L(2,1)-labelings of the triangular lattice. J. Comb. Optim. 29 (2015) 655-669.

[3] B. Dai and W. Lin, On $(s, t)$-relaxed $L(2,1)$-labelings of the hexagonal lattice. ARS Comb. 130 (2017) 319-331.

[4] J. Fiala, T. Kloks and J. Kratochvíl, Fixed-parameter complexity of $\lambda$-labelings. Disc. Appl. Math. 113 (2001) 59-72.

[5] J.R. Griggs and R.K. Yeh, Labelling graphs with a condition at distance 2. SIAM J. Disc. Math. 5 (1992) 586-595.

[6] M.A. Henning and C. Löwnstein, Locating-total domination in claw-free cubic graphs. Disc. Math. 312 (2012) $3107-3116$.

[7] J. Kratochvíl, D. Kratsch and M. Liedloff, Exact algorithms for L(2,1)-labeling of graphs. In Vol. 4708 of Lecture Notes in Computer Science, Springer, Berlin-Heidelberg (2007) 513-524.

[8] W. Lin, On $(s, t)$-relaxed $L(2,1)$-labeling of graphs. J. Comb. Optim. 31 (2014) 1-22.

[9] C. Schwarz and D.S. Troxell, L(2,1)-labelings of products of two cycles. Disc. Appl. Math. 154 (2006) $1522-1540$.

[10] Z. Shao, R.K. Yeh, K.K. Poon and W.C. Shiu, The $L(2,1)$-labeling of $K_{1, n}$-free graphs and its applications. Appl. Math. Lett. 21 (2008) 1188-1193.

[11] Z. Shao, A. Vesel and J. Xu, Frequency assignment problem in networks with limited spectrum. Cent. Eur. J. Oper. Res. 25 (2017) 699-723.

[12] J.J. Sylvester, Mathematical questions with their solutions. Edu. Times 41 (1884) 171-178.

[13] T. Zhao and G. Hu, A note on $(s, t)$-relaxed L(2,1)-labeling of graphs. J. Comb. Optim. 34 (2017) 378-382. 